\title{
Synthesis, Characterization and Antimicrobial Activity of Heterocyclic Azodyes Derived from Thiadiazole
}

\author{
C. T. KEERTHI KUMAR ${ }^{a}$, J. KESHAVAYYA*, T. RAJESH, \\ S. K. PEETHAMBAR ${ }^{\mathrm{b}}$ and R. A. SHOUKAT ALI
}

${ }^{a}$ Department of Studies and Research in Chemistry, School of Chemical Sciences, Kuvempu University, Jnana Sahyadri, Shankaraghatta-577451, Karnataka, India

${ }^{\mathrm{b}}$ Department of Biochemistry, Kuvempu University, Jnana Sahyadri, Shankaraghatta-577451, Karnataka, India

jkeshavayya@gmail.com

Received 6 April 2013 / Accepted 6 May 2013

\begin{abstract}
Ethyl-1,3,4-thiadiazole-2-amine have been synthesized by single step reaction. A series of heterocyclic azodyes were synthesized by coupling 8-hydroxyquinoline, 2,6-diaminopyridine, $\mathrm{N}, \mathrm{N}$-dimethyl aniline, 2-napthol and resorcinol with diazotized 5-ethyl-1,3,4-thiadiazol-2-amine in nitrosyl sulphuric acid. These dyes were characterized by UV, IR, ${ }^{1} \mathrm{H}$ NMR, ${ }^{13} \mathrm{C}$ NMR, elemental analysis and mass spectrometry for selected dyes. The synthesized compounds were also screened for biological activity.
\end{abstract}

Keywords: 5-Ethyl-1,3,4-thiadiazol-2-amine, 8-Hydroxy quinoline, 2,6-Diaminopyridine, $N, N$ dimethyl aniline, 2-Napthol, Azo dyes, Antimicrobial activity

\section{Introduction}

Azo dyes are the most extensively used class of coloring materials because of their massive applications in various fields of science and technology. These are successfully employed as LCD color filters ${ }^{1}$, chromophoric substrates for redox enzymes ${ }^{2}$, optical switches ${ }^{3}$, chemical sensors ${ }^{4}$, textile dyes ${ }^{5}$, lasers ${ }^{6}$, optical data storage ${ }^{7}$, non-linear optics ${ }^{8}$ and also they have advanced applications in organic synthesis ${ }^{9}$. Azo dyes containing sulfur and/or nitrogen atoms have been the subject of many studies recently. These dyes provide bright and strong shades that range from red to green and blue ${ }^{10,11}$. In this regard, a number of studies have been devoted to the characterization, purification and application of azo dyes as antibacterial agents $^{12,13}$. 1,3,4-Thiadiazole derivatives exhibits a broad spectrum of biocidal activities possibly due to the presence of toxophoric-N-C-S moiety ${ }^{14}$.

With these objects in view and also work carried out in our lab on above class of azo dyes $^{15,16}$, we now focused on synthesis and screening for antimicrobial activity of heterocyclic azo dyes. 5-Ethyl-1,3,4-thiadiazole-2-amine was synthesized and was transformed 
to their corresponding diazonium salt by diazotization reaction and were further coupled with various coupling agents (2-naphthol, 8-hydroxy quinolone, 2,6-diaminopyridine, $N, N$-dimethyl aniline and resorcinol) under suitable experimental reaction.

\section{Experimental}

Propionic acid $(0.1 \mathrm{~mol})$ and thiosemicarbazide $(0.1 \mathrm{~mol})$ in conc. sulphuric acid $(20 \mathrm{~mL})$ were refluxed gently for $30 \mathrm{~min}$. The solution was cooled and poured into beaker containing crushed ice. The separated solid was filtered and suspended in water and basified with sodium bicarbonate and kept overnight. The obtained solid was again filtered, washed with water, dried and crystallized from ethanol to obtain a colorless solid with $54 \%$ yield. The general scheme for the synthesis is given in the Scheme 1.<smiles>CCC(=O)O</smiles>
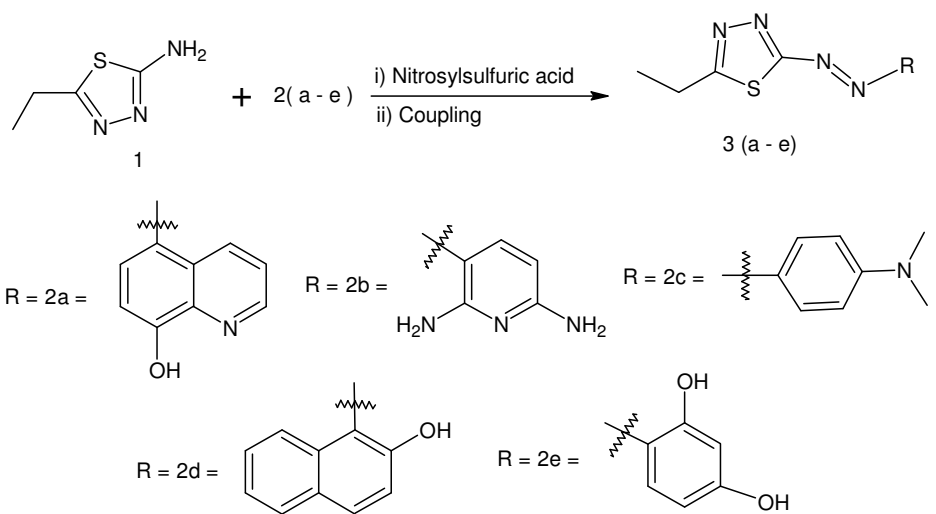

\section{Scheme 1}

\section{General method for synthesis of hetarylazo dye}

5-Ethyl-1,3,4-thiadiazol-2-amine $\left(2.0 \times 10^{-3} \mathrm{~mol}\right)$ was dissolved in hot glacial acetic acidpropionic acid mixture $(2: 1,6.0 \mathrm{~mL})$ and was rapidly cooled in an ice/salt bath to $-5{ }^{\circ} \mathrm{C}$. The liquor was then added in portions during $30 \mathrm{~min}$ to a cold solution of nitrosyl sulphuric acid prepared from sodium nitrite $(0.15 \mathrm{~g})$ and concentrated sulphuric acid $\left(3 \mathrm{~mL}\right.$ at $\left.50{ }^{\circ} \mathrm{C}\right)$. The mixture was stirred for an additional $2 \mathrm{~h}$ at $0{ }^{\circ} \mathrm{C}$. Excess nitrous acid was destroyed by addition of urea. The resulting diazonium salt was cooled in salt/ice mixture. After diazotization was complete the diazo liquor was slowly added to vigorously stirred solution of 8-hydroxyquinoline $\left(2.0 \times 10^{-3} \mathrm{~mol}\right)$ in potassium hydroxide $\left(2.0 \times 10^{-3} \mathrm{~mol}\right)$ and water $(20 \mathrm{~mL})$. The solution was stirred at $0-5{ }^{0} \mathrm{C}$ for $2 \mathrm{~h}$. After $2 \mathrm{~h}$, the $\mathrm{pH}$ of the reaction mixture was maintained at 4-6 by the simultaneous addition of saturated sodium carbonate solution. The mixture was stirred for one day at room temperature. After one day, the resulting solid was filtered, washed with cold water and dried.

\section{Preparation of 5-(5-ethyl-2-thiadiazolylazo)-8-hydroxyquinoline (3a)}

This dye was synthesized from 5-ethyl-1,3,4-thiadiazol-2-amine and 8-hydroxyquinoline as brown crystals (yield- 50\%; m.p: $222{ }^{0} \mathrm{C}$ ). IR [(KBr) $\left.v_{\max } / \mathrm{cm}^{-1}\right]$ : 3428-3268 (quinoline- $\mathrm{OH}$ ), 
3075 (aromatic C-H), 2973 (aliphatic C-H), $1554(\mathrm{C}=\mathrm{N}), 1420(\mathrm{~N}=\mathrm{N}), 1208(\mathrm{C}-\mathrm{O}) \mathrm{cm}^{-1} ;{ }^{1} \mathrm{H}$ NMR (DMSO-d $\left.)_{6}\right): 8.8(\mathrm{~d}, 1 \mathrm{H}), 8.6(\mathrm{~d}, 1 \mathrm{H}), 7.8(\mathrm{t}, 1 \mathrm{H}), 6.7(\mathrm{~d}, 1 \mathrm{H}), 7.2(\mathrm{~d}, 1 \mathrm{H}), 3.1(\mathrm{q}, 2 \mathrm{H})$, $1.4(\mathrm{t}, 3 \mathrm{H}) .{ }^{13} \mathrm{C}$ NMR (DMSO-d 6 , ppm): $14.3\left(\mathrm{CH}_{3}\right), 24.5\left(\mathrm{CH}_{2}\right), 153.2(\mathrm{C}-\mathrm{O}), 137.7$ $(\mathrm{C}=\mathrm{N}), 130.7(\mathrm{C}-\mathrm{N})$, LC-MS: $(\mathrm{M}+1)^{+}(\mathrm{m} / \mathrm{z}): 286(19.8 \%), 172(21.2 \%), 144(100 \%)$. Anal. Calcd. For $\mathrm{C}_{13} \mathrm{H}_{11} \mathrm{~N}_{5} \mathrm{OS}$ : C,54.72; H, 3.89; N, 24.55; found: C, 54.70; H, 3.86; N, 24.52.

\section{Preparation of 3-[(5-ethyl-1, 3, 4-thiadiazol-2-yl)diazenyl]pyridine-2,6-diamine (3b)}

This dye was prepared from 5-ethyl-1,3,4-thiadiazol-2-amine and 2,6-diamino pyridine as wine red crystals (yield:62\%; m.p: $202{ }^{0} \mathrm{C}$ ). IR [(KBr) $\left.v_{\max } / \mathrm{cm}^{-1}\right]: 3342 \mathrm{~cm}^{-1}, 3227 \mathrm{~cm}^{-1}$ (N-H stretch), 2965 (aliphatic C-H), $1639 \mathrm{~cm}^{-1}$ (N-H aromatic) $1427 \mathrm{~cm}^{-1}$ (N=N stretch); ${ }^{1} \mathrm{H}$ NMR (DMSO-d 6 ): 7.7 (d, 1H), $6.3(\mathrm{~d}, 1 \mathrm{H}), 3.0(\mathrm{q}, 2 \mathrm{H}), 1.35(\mathrm{t}, 3 \mathrm{H}) .,{ }^{13} \mathrm{C}$ NMR (DMSO$\left.\mathrm{d}_{6}, \mathrm{ppm}\right)$ : $14.9\left(\mathrm{CH}_{3}\right), 24.9\left(\mathrm{CH}_{2}\right), 157.8(\mathrm{C}-\mathrm{N}), 169.2(\mathrm{C}=\mathrm{N}), 158.9(\mathrm{C}-\mathrm{N})$, Anal. Calcd. For $\mathrm{C}_{9} \mathrm{H}_{11} \mathrm{~N}_{7} \mathrm{~S}: \mathrm{C}, 43.36 ; \mathrm{H}, 4.45 ; \mathrm{N}, 39.33$; found: $\mathrm{C}, 43.34 ; \mathrm{H}, 4.42 ; \mathrm{N}, 39.31 \%$.

Preparation of 4-[(5-ethyl-1, 3,4-thiadiazol-2-yl) diazenyl]-N,N-dimethyl aniline (3c)

This dye was obtained from 5-ethyl-1,3,4-thiadiazol-2-amine and $N, N$-dimethylaniline as dark red crystals (yield-71\% , m.p:198). IR [(KBr) $\left.v_{\max } / \mathrm{cm}^{-1}\right]$ : 3015 (aromatic C-H), 2939 (aliphatic C-H), $1546(\mathrm{C}=\mathrm{N}) \mathrm{cm}^{-1} ; 1435(\mathrm{~N}=\mathrm{N}) \mathrm{cm}^{-1}$; ${ }^{1} \mathrm{H}$ NMR (DMSO-d $\left.{ }_{6}\right): 7.2(\mathrm{~d}, 2 \mathrm{H}), 6.7$ $(\mathrm{d}, 2 \mathrm{H}), 2.85(\mathrm{~s}, 6 \mathrm{H}), 3.1(\mathrm{q}, 2 \mathrm{H}), 1.4(\mathrm{t}, 3 \mathrm{H}) .,{ }^{13} \mathrm{C}$ NMR (DMSO-d 6 , ppm): 14.9 $\left(\mathrm{CH}_{3}\right)$, 25.1 $\left(\mathrm{CH}_{2}\right), 40.7\left(\mathrm{CH}_{3}\right), 170.1(\mathrm{C}=\mathrm{N}), 150.5(\mathrm{C}-\mathrm{N})$, Anal. Calcd. For $\mathrm{C}_{12} \mathrm{H}_{15} \mathrm{~N}_{5} \mathrm{~S}: \mathrm{C}, 55.15 ; \mathrm{H}$, $5.79 ; \mathrm{N}, 26.80$; found: $\mathrm{C}, 55.13 ; \mathrm{H}, 5.77 ; \mathrm{N}, 26.78$.

\section{Preparation of 1-[(5-ethyl-1, 3, 4-thiadiazol-2-yl)diazenyl]naphthalen-2-ol (3d)}

This dye was synthesized from 5-ethyl-1,3,4-thiadiazol-2-amine and 2-naphthol as brick red crystals (yield-66\% , m.p:219). IR [(KBr) $\left.v_{\max } / \mathrm{cm}^{-1}\right]: 3432-3215(-\mathrm{OH}), 2965$ (aliphatic $\mathrm{C}-\mathrm{H}), 1542(\mathrm{C}=\mathrm{N}), 1432(\mathrm{~N}=\mathrm{N}),{ }^{1} \mathrm{H}$ NMR (DMSO-d $\left.\mathrm{d}_{6}\right): 7.4-7.6(\mathrm{~m}, 3 \mathrm{H}), 7.0-7.2(\mathrm{~m}, 3 \mathrm{H}), 2.9$ $(\mathrm{q}, 2 \mathrm{H}), 1.3(\mathrm{t}, 3 \mathrm{H}) .{ }^{13} \mathrm{C}$ NMR (DMSO-d $\left.\mathrm{d}_{6}, \mathrm{ppm}\right): 15.1\left(\mathrm{CH}_{3}\right), 25.4\left(\mathrm{CH}_{2}\right), 169.3(\mathrm{C}=\mathrm{N})$, 163.1(C-O), 135.6(C-N). Anal. Calcd. For $\mathrm{C}_{14} \mathrm{H}_{12} \mathrm{~N}_{4} \mathrm{OS}$ : C,59.14; H, 4.25;N, 19.70; found: C, 59.11; H, 4.22; N, $19.68 \%$.

\section{4-[(5-Ethyl-1,3,4-thiadiazol-2-yl)diazenyl]benzene-1,3-diol (3e)}

This dye was prepared from 5-ethyl-1,3,4-thiadiazol-2-amine and resorcinol as red crystals (yield-64\% , m.p:189). IR [(KBr) $\left.v_{\max } / \mathrm{cm}^{-1}\right]:$ 3428-3248 (-OH), 2965 (aliphatic C-H), 1548 $(\mathrm{C}=\mathrm{N}), 1439(\mathrm{~N}=\mathrm{N}),{ }^{1} \mathrm{H}$ NMR $\left(\mathrm{DMSO}_{6}\right): 6.4-6.8(\mathrm{dd}, 2 \mathrm{H}), 6.2(\mathrm{~s}, \mathrm{H}), 2.9(\mathrm{q}, 2 \mathrm{H}), 1.4$ $(\mathrm{t}, 3 \mathrm{H}) .,{ }^{13} \mathrm{C}$ NMR (DMSO-d 6 , ppm):14.7 $\left(\mathrm{CH}_{3}\right), 24.4\left(\mathrm{CH}_{2}\right), 168.5(\mathrm{C}=\mathrm{N}), 160.1(\mathrm{C}-\mathrm{O})$. Anal. Calcd. For $\mathrm{C}_{10} \mathrm{H}_{10} \mathrm{~N}_{4} \mathrm{O}_{2} \mathrm{~S}$ : C,47.99; H, 4.03; N, 22.39; found: C, 48.03; H, 4.07; N, $22.42 \%$.

\section{Biological activity}

\section{Antibacterial and antifungal activity}

The antimicrobial activity of newly synthesized compounds was evaluated using agar disc diffusion assay ${ }^{17-18}$. Briefly, a 24 hours old culture of bacteria and 48 hours old culture of fungi was mixed with sterile physiological saline $(0.9 \%)$ and the turbidity was adjusted to the standard inoculum of MacFarland scale $0.5\left(10^{6}\right.$ colony forming units (CFU) per $\left.\mathrm{mL}\right)$. Petri plates containing $20 \mathrm{~mL}$ of Mueller Hinton Agar and Sabouraud-dextrose agar was used for antibacterial and antifungal activity. The inoculums was spread on the surface of the solidified media and Whatman No. 1 filter paper discs $(5 \mathrm{~mm}$ in diameter) impregnated with the test compound $(20 \mu \mathrm{L} / \mathrm{disc})$ were placed on the solidified media. Streptomycin $(5 \mathrm{mg} / \mathrm{disc})$ and fluconazole $(5 \mathrm{mg} / \mathrm{disc})$ were used as positive control for bacteria and fungi respectively 
along with DMSO disc as negative control. Zone of inhibition was recorded in millimeters after incubating bacterial strains at $37{ }^{\circ} \mathrm{C}(24 \mathrm{~h})$ and fungal strains at $25^{\circ} \mathrm{C}(72 \mathrm{~h})$. Tests were performed in triplicate and the values were expressed as mean $\pm \mathrm{SD}^{19-20}$.

\section{Results and Discussion}

As shown in Scheme 1 the hetarylazo dyes 3(a-e) were prepared through the diazotization of 5-ethyl-1,3,4-thiadiazol-2-amine and coupled with different coupling components 2-napthol, 8-hydroxyquinoline, 2,6-diaminopyridine $N, N$ dimethyl aniline and resorcinol. These dyes were in good yield and these are soluble in acetone, DMF and DMSO. The yield, melting point, $\lambda_{\max }$, molar absorptivity $(\varepsilon)$, molecular formula and solubility data of all synthesized dyes are tabulated in Table 1. Absorption spectra of the azo dyes 3(a-e) were recorded in DMSO at a concentration of $10^{-6} \mathrm{~mol} \mathrm{~L}^{-1}$. The results are summarized in Figure 1. The infrared spectra of all the dyes (in $\mathrm{KBr}$ ) for the dyes 3a, 3d and 3e a broad band has appeared at 3500-3200 which confirms the presence of hydroxyl group (-OH). The dyes 3(a-e) are showed $1417-1435 \mathrm{~cm}^{-1}$ for $(\mathrm{N}=\mathrm{N})$ group. The $v_{\max }$ values at $3085-3005 \mathrm{~cm}^{-1}$ (aromatic $\mathrm{C}-\mathrm{H}$ ) and at $2986-2851 \mathrm{~cm}^{-1}$ (aliphatic C-H) were also observed.

Table 1. Yield, melting point, $\lambda_{\max }$, molar absorptivity $(\varepsilon)$, molecular formula and solubility data of dyes 3(a-e)

\begin{tabular}{|c|c|c|c|c|c|c|c|}
\hline Dye & $\begin{array}{c}\text { Yield, } \\
\%\end{array}$ & $\begin{array}{l}\text { M.P, } \\
{ }^{0} \mathrm{C}\end{array}$ & $\begin{array}{c}\lambda_{\max } \text { in } \\
n m\end{array}$ & $\log \varepsilon$ & $\begin{array}{l}\text { Molecular } \\
\text { formula }\end{array}$ & $\begin{array}{c}\text { Mol. } \\
\text { wt }\end{array}$ & Solubility \\
\hline $3 a$ & 50 & $220-222$ & 522 & 4.47 & $\mathrm{C}_{13} \mathrm{H}_{11} \mathrm{~N}_{5} \mathrm{OS}$ & 285.32 & $\begin{array}{l}\text { Acetone / DMF } \\
\text { Ethanol /DMSO }\end{array}$ \\
\hline $3 b$ & 62 & $199-202$ & 515 & 4.29 & $\mathrm{C}_{9} \mathrm{H}_{11} \mathrm{~N}_{7} \mathrm{~S}$ & 249.29 & $\begin{array}{l}\text { Acetone/DMSO } \\
\text { DMF/Methanol }\end{array}$ \\
\hline $3 c$ & 71 & 195-198 & 513 & 4.68 & $\mathrm{C}_{12} \mathrm{H}_{15} \mathrm{~N}_{5} \mathrm{~S}$ & 261.34 & $\begin{array}{l}\text { Acetone/ DMSO } \\
\text { Methanol/DMF }\end{array}$ \\
\hline $3 d$ & 66 & $215-219$ & 509 & 4.09 & $\mathrm{C}_{14} \mathrm{H}_{12} \mathrm{~N}_{4} \mathrm{OS}$ & 284.33 & $\begin{array}{l}\text { Acetone/ DMSO } \\
\text { Methanol/DMF }\end{array}$ \\
\hline $3 e$ & 64 & $188-190$ & 517 & 4.23 & $\mathrm{C}_{10} \mathrm{H}_{10} \mathrm{~N}_{4} \mathrm{O}_{2} \mathrm{~S}$ & 250.27 & $\begin{array}{l}\text { Acetone/ DMSO } \\
\text { Methanol/DMF }\end{array}$ \\
\hline & & & & & & $\begin{array}{r}3 \mathrm{a} \\
3 \mathrm{~b} \\
3 \mathrm{c} \\
3 \mathrm{~d} \\
\mathrm{e} \\
\end{array}$ & \\
\hline & & & & & & & \\
\hline & & 300 & & & 500 & 600 & \\
\hline
\end{tabular}

Figure 1. Absorption spectra of dyes 3(a-e) in Acetone 
The ${ }^{1} \mathrm{H}$ NMR spectra was recorded in DMSO- $\mathrm{d}_{6}$ at room temperature showed a quadrate at 3.0-3.2 ppm $\left(\mathrm{CH}_{2}\right)$, a triplet at 1.4- $1.85 \mathrm{ppm}\left(\mathrm{CH}_{3}\right)$ for the dyes $\mathbf{3}(\mathbf{a}-\mathbf{e})$, in dye 3c singlet at $2.85 \mathrm{ppm}$ are for $-\left(\mathrm{CH}_{3}\right)_{2}$ group of $N, N$-dimethyl aniline, a multiplet from 7.20 to $7.50 \mathrm{ppm}$ for aromatic protons (Aro-H).

\section{Biological activity}

Synthesized organic compounds were evaluated for the antimicrobial activity with standard drugs (Streptomycin and fluconazole). The closer look into the biological studies of these synthesized dyes revealed that compound $\mathbf{3 a}$ and $\mathbf{3 b}$ showed much better activities when compare to the other compounds. The results from the antimicrobial activity of synthesized organic compounds (Table 2) prompted us to investigate their antifungal activity (Table 3) against important pathogens. Although a comparable antibacterial activity was exhibited by few compounds, these compounds failed to show a good response to antifungal activity and the compound 3c doesn't show any antimicrobial property.

Table 2. In vitro antibacterial activities of the compounds 3(a-e)

\begin{tabular}{|c|c|c|c|c|c|c|c|}
\hline \multirow{3}{*}{ S.No. } & \multirow[t]{2}{*}{ Compounds } & \multicolumn{2}{|c|}{$\begin{array}{l}\text { Escherichia } \\
\text { coli }\end{array}$} & \multicolumn{2}{|c|}{$\begin{array}{c}\text { Staphylococcus } \\
\text { aureus }\end{array}$} & \multicolumn{2}{|c|}{$\begin{array}{c}\text { Pseudomonas } \\
\text { aeruginosa }\end{array}$} \\
\hline & & \multicolumn{6}{|c|}{ Diameter of zone of inhibition, $\mathrm{mm}$} \\
\hline & $\begin{array}{l}\text { Conc. in } \\
\mathrm{mg} / \mathrm{mL}\end{array}$ & 1 & 0.5 & 1 & 0.5 & 1 & 0.5 \\
\hline 1 & Control & 0 & & 0 & & 0 & \\
\hline 2 & $\begin{array}{c}\text { Standard } \\
\text { Streptomycin }\end{array}$ & $16 \pm 0.4$ & $10 \pm 0.5$ & $15 \pm 0.4$ & $10 \pm 0.4$ & $16 \pm 0.5$ & $13 \pm 0.4$ \\
\hline 3 & 3a & $8 \pm 0.4$ & $4 \pm 0.5$ & $7 \pm 0.5$ & $4 \pm 0.6$ & $8 \pm 0.4$ & $4 \pm 0.5$ \\
\hline 4 & $3 b$ & $0.7 \pm 0.4$ & $4 \pm 0.6$ & $6 \pm 0.6$ & $4 \pm 0.5$ & $7 \pm 0.5$ & $4 \pm 0.4$ \\
\hline 5 & $3 c$ & $2 \pm 0.5$ & $2 \pm 0.5$ & $2 \pm 0.6$ & $2 \pm 0.5$ & $2 \pm 0.4$ & $2 \pm 0.4$ \\
\hline 6 & 3d & $2 \pm 0.4$ & $2 \pm 0.5$ & $2 \pm 0.5$ & $2 \pm 0.5$ & $2 \pm 0.4$ & $2 \pm 0.5$ \\
\hline 7 & $3 e$ & $2 \pm 0.4$ & $2 \pm 0.5$ & $2 \pm 0.6$ & $2 \pm 0.4$ & $2 \pm 0.4$ & $2 \pm 0.5$ \\
\hline
\end{tabular}

Table 3. In vitro antifungal activities of the compounds 3(a-e)

\begin{tabular}{cccccccc}
\hline & \multirow{2}{*}{ S.No. } & \multicolumn{2}{c}{$\begin{array}{c}\text { Aspergillus } \\
\text { Flavus }\end{array}$} & \multicolumn{3}{c}{$\begin{array}{c}\text { Chrysosporium } \\
\text { Keratinophilum }\end{array}$} & \multicolumn{2}{c}{$\begin{array}{c}\text { Candida } \\
\text { Albicans }\end{array}$} \\
\cline { 2 - 8 } & & \multicolumn{3}{c}{ Diameter of zone of inhibition, mm } \\
\cline { 2 - 8 } & $\begin{array}{c}\text { Conc. in } \\
\text { mg/mL }\end{array}$ & 1 & 0.5 & 1 & 0.5 & 1 & 0.5 \\
\hline 1 & Control & 0 & & 0 & & 0 & \\
2 & Standard & $13 \pm 0.4$ & $10 \pm 0.6$ & $17 \pm 0.4$ & $15 \pm 0.5$ & $22 \pm 0.5$ & $20 \pm 0.4$ \\
3 & Streptomycin & & & & & & \\
4 & 3a & $6 \pm 0.6$ & $3 \pm 0.5$ & $7 \pm 0.4$ & $4 \pm 0.6$ & $5 \pm 0.5$ & $2 \pm 0.6$ \\
5 & 3b & $2 \pm 0.5$ & $1 \pm 0.4$ & $2 \pm 0.6$ & $1 \pm 0.5$ & $2 \pm 0.6$ & $1 \pm 0.5$ \\
6 & 3c & 0 & 0 & 0 & 0 & 0 & 0 \\
7 & 3d & $2 \pm 0.6$ & $1 \pm 0.4$ & $2 \pm 0.6$ & $1 \pm 0.5$ & $2 \pm 0.6$ & $1 \pm 0.5$ \\
\hline
\end{tabular}

\section{Conclusion}

In this work, 5 new heterocyclic azo dyes were synthesized by a classical method of diazotizing-coupling. Their structures were confirmed by ${ }^{1} \mathrm{H}$ and ${ }^{13} \mathrm{C}$ NMR, FT-IR and UV-Vis spectra. All the dyes tested had some effect on bacterial growth, most potency being shown with dye number $\mathbf{3 a}$ and $\mathbf{3 b}$. Dye 3a is shown the good antifungal activity. 


\section{References}

1. Sakong C, Kim Y D, Choi J H, Yoon C and Kim J P, Dyes and Pigments, 2011, 88(2), 166-173.

2. Haghbeen K and Tan W E, J Org Chem., 1998, 63, 4503-4505.

3. Coelho P J, Castro M C R, Fonseca A M C and Raposo M M M, Dyes and Pigments, 2011, 92(1), 745-748.

4. Lee H Y, Song X, Park H, Baik M H and Lee D, J Am Chem Soc., 2010, 132(38), 12133-12144.

5. Burkinshaw S M and Paraskevas M, Dyes and Pigments, 2011, 88(3), 396-402.

6. Gayathri C and Ramalingam A, Optik., 2008, 119, 409-414.

7. Lutfor M R, Hegde G, Kumar S, Tschierske C and Chigrinov V G, Opt Mater., 2009, 32, 176-183.

8. Raposo M M M, Castro M C R, Fonseca A M C, Schellenberg P and Belsley M, Tetrahedron, 2011, 67, 5189-5198.

9. Kawasaki M and Yamamoto H, J Am Chem Soc., 2006, 128(51), 16482-16483.

10. Maradiya H R and Patel V S, Fibers Polymer, 2001, 2(3), 153-158.

11. Seferoglu Z, Tokay N, Hokelek T and Sahin E, Struct Chem., 2008, 19, 559-564.

12. Manojkumar P, Ravi T K and Gopalakrishnan S, Eur J Med Chem., 2009, 44, 4690-4694.

13. Yousefi H, Yahyazadeh A, Moradi Rufchahi E O and Rassa M, J Mol Liq., 2013, 180, 51-58.

14. Mavrova A, Wesselinova D, Tsenov Y A and Denkova P, Eur J Med Chem., 2009, 44, 63-69.

15. Keerthi Kumar C T, Keshavayya J, Rajesh T and Peethambar S K, Int J Pharm Pharm Sci., 2013, 5, (Suppl 1), 296-301.

16. Shridhar A H, Keshavayya J, Peethambar S K and Joy H Hoskeri, Arabian J Chem., 2012; DOI:10.1016/j.arabjc.2012.04.018.

17. Arthington-Skaggs B A, Motley M, Warnock D W and Morrison C J, J Clin Microbiol., 2000, 38, 2254-2260.

18. Vijesh A M, Arun M Isloor, Sandeep Telkar, Peethambar S K, Sankappa Rai, Nishitha Isloor, Eur J Med Chem., 2009, 44, 3350-3355.

19. Rocha L, Marston A, Potterat O, Kaplan M A C, Stoeckli-Evans H and Hostettmann K, Phytochem., 1995, 40(5), 1447-1452.

20. Mac Lowry D J, Jaqua M J and Selepak S T, Appl Microbiol., 1970, 20, 46-53. 